\title{
Cutaneous Elastorrhexis; A Heralding Sign of Gronblad-Strandberg Syndrome: A Case Report
}

\author{
Ravindranath Brahmadeo Chavan ${ }^{\# 1}$, Nitika S Deshmukh (iD) ${ }^{\# 1}$, Vasudha Abhijit Belgaumkar ${ }^{1,{ }^{*}}$ and \\ Vijay Raut $^{1}$ \\ ${ }^{1}$ Departement of Skin VD, BJGMC, Pune, India \\ "Corresponding author: Associate Professor, Departement of Skin VD, BJGMC, Pune, India. Email: doc_vasudha@yahoo.co.in \\ \# These authors are contributed equally as the first author. \\ Received 2020 May 26; Revised 2020 June 13; Accepted 2020 June 20.
}

\begin{abstract}
Introduction: Pseudoxanthoma Elasticum (PXE), also called Gronblad-Strandberg syndrome, is an autosomal recessive disorder due to mutation in the ABCC6 allele on chromosome 16p. It is characterized by the progressive fragmentation and calcification of elastin fibers of the dermis, blood vessels, and Bruch's membrane of the eye. In this article, the authors present a case of PXE with cutaneous elastorrhexis.

Case Presentation: A 31-year-old female presented with skin changes in the form of yellowish linearly arranged papules over the lateral side of the neck and anterior abdomen since adolescence. Her retinoscopy and cardiovascular examinations were reported as normal. The molecular analysis could not be done due to financial limitations. Based on these findings, the patient was suspected of PXE according to the revised criteria for the diagnosis of PXE.

Conclusions: Skin lesions are generally first to appear in adolescence. Ocular findings develop at later ages, i.e., third or fourth decades. Cardiovascular manifestations develop later in life. Hence, skin changes can aid in the early diagnosis of PXE and help clinicians to screen patients for systemic complications. Being a multisystem heritable disorder with morbidity and mortality, there is a need to formulate the clinical criteria for definitive diagnosis in resource-poor settings where molecular assays cannot be performed. The recognition of typical skin lesions can aid in the accurate diagnosis to facilitate the early detection and management of life-threatening systemic complications.
\end{abstract}

Keywords: Cutaneous Elastorrhexis, Gronblad-Strandberg Syndrome

\section{Introduction}

Pseudoxanthoma Elasticum (PXE), also called Gronblad-Strandberg syndrome, is an inherited multisystem disorder characterized by the progressive fragmentation and calcification of dermal and vascular elastic fibers and Bruch's membrane of the eye. Globally, it affects one in every 50,000 population with a slight female predominance (1). We report a case of probable PXE with isolated cutaneous lesions.

\section{Case Presentation}

A 31-year-old female presented with asymptomatic lesions over the neck and abdomen since adolescence. She had no history of hematemesis, malena, or blood in the stool. She denied a history of ischemic heart disease, claudication, or diminution of vision. There was no history of any complaints in other family members. On examination, there were multiple small yellowish papules closely arranged in a reticulate pattern with cobblestone or plucked chicken skin-like appearance over the neck and abdomen (Figure 1). Mucosal and palmoplantar examinations were normal, with no lesions elsewhere on the body. The differential diagnosis of PXE and PXE-like disorders were considered. Laboratory investigations (hemogram, liver and renal function tests, serum calcium, and phosphate levels) were within normal limits. Electrocardiography, 2D Echo, and ocular examination (retinoscopy) were unremarkable. Histopathology of skin lesions revealed clumped, degenerated, and fragmented elastic fibers in the mid dermis, which was suggestive of pseudoxanthoma elasticum (Figures 2 and 3). Few elastic fibers and collagen fibers showed calcification with von Kossa stain (Figure 4). Molecular analysis of gene ABCC6 could not be performed due to the lack of facilities. 

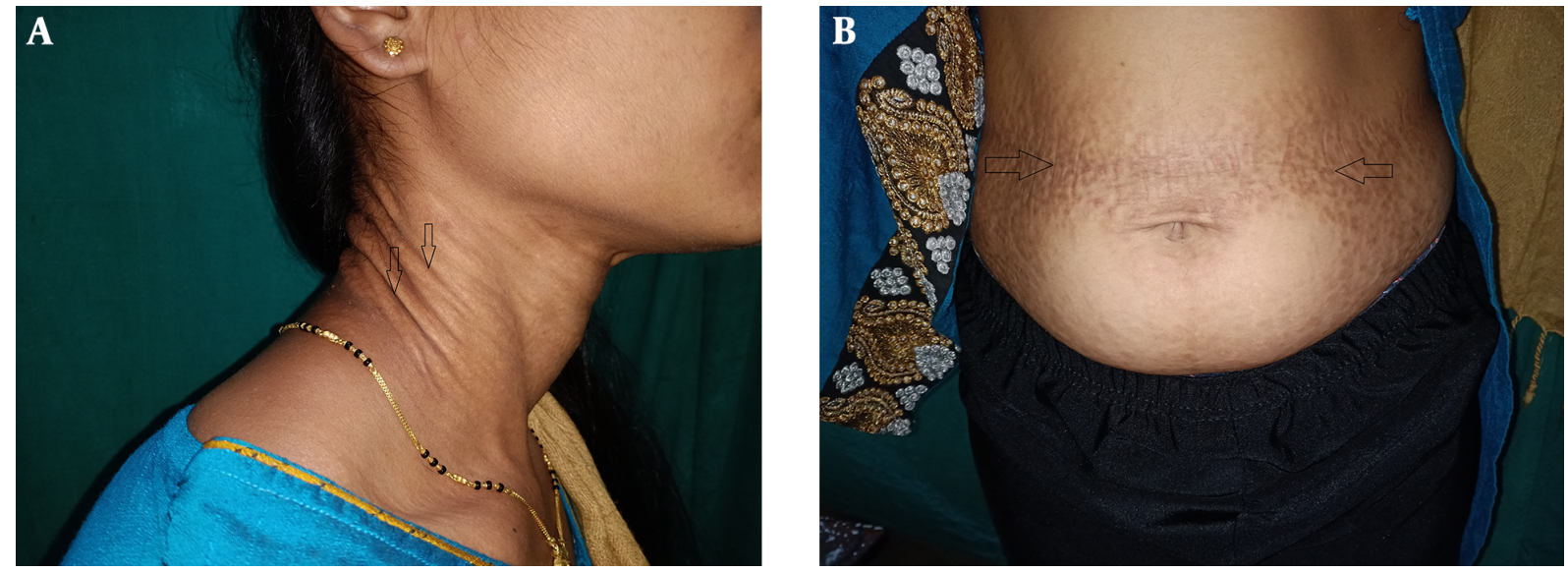

Figure 1. A, Moroccan leather appearance of the lateral side of the neck; B, Moroccan leather appearance of the anterior abdomen

A probable diagnosis of pseudoxanthoma elasticum (type II) was made based on the clinical and histopathological findings. The patient was counseled regarding the course and prognosis of the disorder and advised strict six-monthly follow-ups to monitor for the appearance of any ocular or cardiovascular manifestations. In addition, we offered fractional $\mathrm{CO}_{2}$ laser treatment for cosmetic improvement of skin lesions, but the patient has not been followed up so far.

\section{Discussion}

Pseudoxanthomaelasticum (systemic elastorrhexis) is a rare genetic disorder with either autosomal recessive or dominant inheritance caused by the mutation of the ABCC6 gene located on chromosome 16p. This gene is a member of the ATP-binding cassette protein, which acts as a transmembrane transporter of anionic peptides. PXE is associated with significant diversity concerning incipience, extent, and magnitude of organ system involvement. Classical cutaneous lesions appear in the form of yellowish papules arranged in a reticulate manner imparting a Moroccan leather or plucked chicken skin appearance. These lesions are prominently located on the neckline, abdomen, axillae, below clavicles, groins, or thighs. The skin lesions generally appear in adolescence and adulthood, but rarely can also appear in old ages or childhood (younger than 10 years). Similar changes might be present over oral and genital mucosa (2).

Organs like eyes and blood vessels mainly composed of elastic fibers, are predominantly involved, resulting in systemic abnormalities. Cardiovascular changes generally manifest in late adulthood. These include the calcification of mesenteric, coronary, and visceral large arteries. Angiography features comprise stenosis or occlusion of peripheral or visceral arteries, angiomatous malformations, and aneurysms. Rarely, cerebral hemorrhage or ischemic heart disease may occur as severe complications.

Ocular changes like yellowish specks in retinal pigment epithelium (peau d'orange fundus) are the earliest ophthalmological signs, usually seen during the first few years of life. Pearly white drusen, punched-out atrophic areas, and leopard spotting may precede the onset of angioid streaks on the retina, which occurs between the ages of 20 and 40 years. However, our patient did not demonstrate any abnormal retinoscopic features during her current visit. PXE patients may also have gastrointestinal (GI) changes in the form of the fragility of submucosal vessels, which may manifest as GI bleeds. Other rare manifestations include recurrent miscarriages, hypercalcemia, hyperphosphatemia, and abnormalities in vitamin D metabolism.

According to the revised criteria for the diagnosis of PXE (3, 4), our patient was diagnosed as a probable case of pseudoxanthoma elasticum, as she met both major criteria pertaining to skin (clinical features of yellowish papules and/or plaques on neck or flexures) and histopathological evidence of the increased amount of altered elastin with clumping, fragmentation, or calcification. The diagnosis would have been further validated on performing the genetic assay of the ABCC6 allele. Various systemic and dermatologic disorders and non-ABCC6 genetic mutations (elastosis, beta-thalassemia, cutis laxa, Paget's disease) may present with manifestations clinically and histopathologically simulating typical PXE. These conditions are associated with cutaneous, ocular, and cardio- 

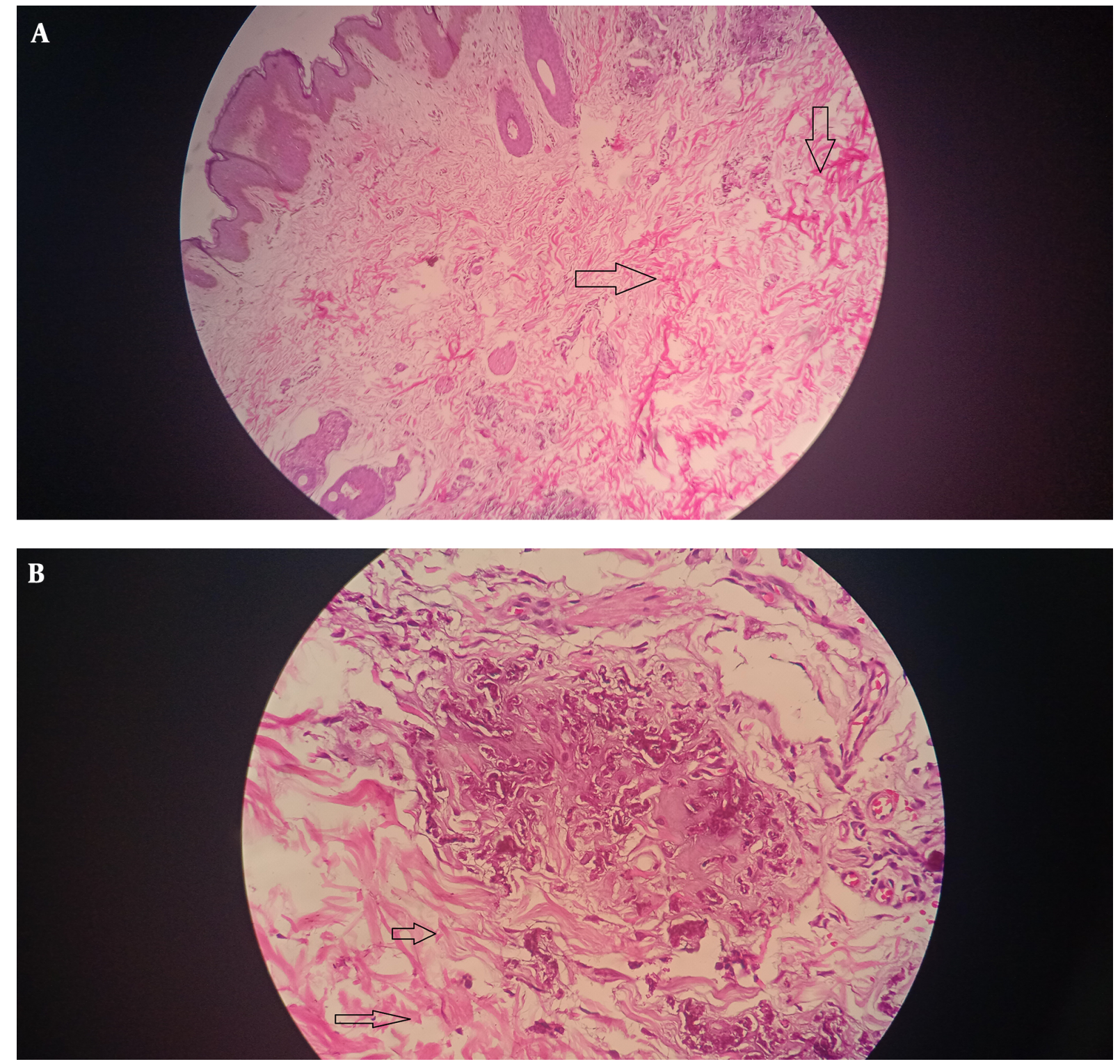

Figure 2. A, Hematoxylin and eosin stain: 10x view showing fragmented elastin fibers in the mid dermis. B, Hematoxylin and eosin stain: 40x view showing fragmented elastin fibers in the mid dermis

vascular alterations termed as PXE-like syndrome and need to be ruled out $(5,6)$.

PXE can occur in the absence of skin involvement. Gastrointestinal involvement frequently occurs in the classic recessive PXE type I variant, while visceral manifestations are minimal or absent (as in the present case) in the type II form (7) Interestingly, despite being in the fourth decade of life, our patient had isolated cutaneous findings, whereas most patients are expected to demonstrate some systemic involvement before they reach this age. The diagnosis of
PXE may be delayed since the manifestations are either not noticeable or not severe enough to warrant medical attention. As skin manifestations precede the onset of ophthalmological and cardiovascular changes, their presence should alert the treating physician to look for the involvement of other systems by regular follow-ups. Surgical management for cosmetic correction is one of the few therapeutic options in the absence of any specific treatment for the cutaneous or systemic involvement. The institution of a low-calcium diet and oral phosphate binders 


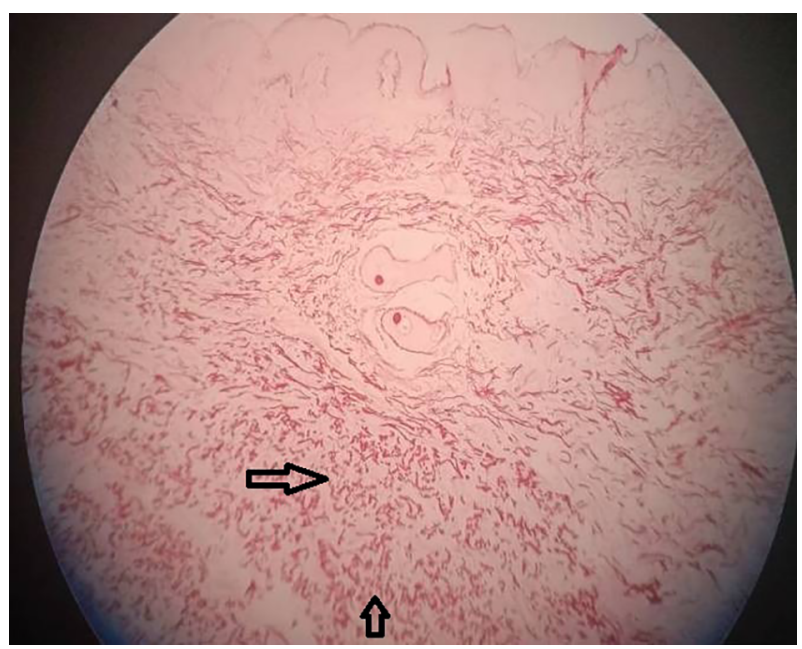

Figure 3. Orcein stain: 10x view showing fragmented elastin fibers in the mid dermis (elastorrhexis)

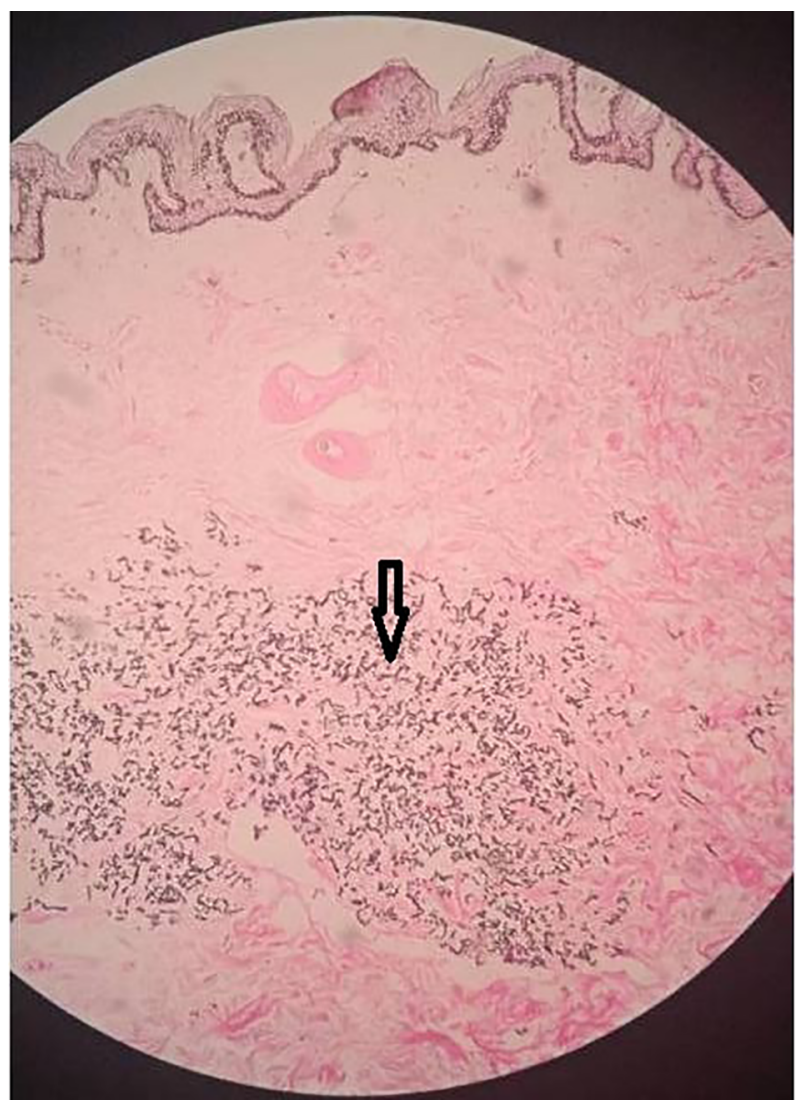

Figure 4. Von Kossa stain: 10x view showing calcification of elastin fibers in the mid dermis has been shown to produce clinical, histopathological, and electron-microscopic improvement (8).

Our patient is being monitored six-monthly for ophthalmological, cardiovascular, and gastrointestinal evaluation.

\subsection{Conclusions}

Diagnosis of pseudoxanthoma elasticum may be missed due to the inconspicuous appearance of skin lesions. As this is a multisystem heritable disorder with morbidity and mortality, there is a need to re-formulate clinical criteria for definitive diagnosis in resource-poor settings where molecular assays cannot be performed. Recognition of typical skin lesions can aid in the accurate diagnosis, which would facilitate the early detection and management of life-threatening systemic complications.

\section{Footnotes}

Authors' Contribution: Study concept and design: Authors 1, 2,3,4, Acquisition of data: Authors 1, 2,3, Analysis and interpretation of data: Author 1,2,3,4, Drafting of the manuscript: Author 1,2,3,4, Critical revision of the manuscript for important intellectual content: Author 1,2,3, Statistical analysis: Author 1,2,3, Administrative, technical, and material support: Author 1,2,3, Study supervision: Author 1,2,3.

Conflict of Interests: There are no conflicts of interest. Funding/Support: No funding or financial support is reported.

Informed Consent: The informed consent form was signed by the patient.

\section{References}

1. Burrows N. Rook's Textbook of Dermatology, Ninth Edition. 2016.

2. Uitto J, Jiang Q, Varadi A, Bercovitch LG, Terry SF. Pseudoxanthoma Elasticum: Diagnostic Features, Classification, and Treatment Options. Expert Opin Orphan Drugs. 2014;2(6):567-77. doi 10.1517/21678707.2014.908702. [PubMed: 25383264]. [PubMed Central: PMC4219573].

3. Germain DP. Pseudoxanthoma elasticum. Orphanet $J$ Rare Dis. 2017;12(1):85. doi: 10.1186/s13023-017-0639-8. [PubMed: 28486967]. [PubMed Central: PMC5424392].

4. Plomp AS, Toonstra J, Bergen AA, van Dijk MR, de Jong PT. Proposal for updating the pseudoxanthoma elasticum classification system and a review of the clinical findings. Am JMed Genet A. 2010;152A(4):1049-58. doi: 10.1002/ajmg.a.33329. [PubMed: 20358627].

5. Finger RP, Charbel Issa P, Ladewig MS, Gotting C, Szliska C, Scholl HP, et al. Pseudoxanthoma elasticum: genetics, clinical manifestations and therapeutic approaches. Surv Ophthalmol. 2009;54(2):272-85. doi: 10.1016/j.survophthal.2008.12.006. [PubMed: 19298904]. 
6. Plomp AS, Bergen AA, Florijn RJ, Terry SF, Toonstra J, van Dijk $\mathrm{MR}$, et al. Pseudoxanthoma elasticum: Wide phenotypic variation in homozygotes and no signs in heterozygotes for the c.3775delT mutation in ABCC6. Genet Med. 2009;11(12):852-8. doi: 10.1097/GIM.ob013e3181c00a96. [PubMed: 19904211].

7. Gregory B, Ho VC. Cutaneous manifestations of gastrointestinal disorders. Part II. Journal of the American Academy of Dermatology.
1992;26(3):371-83.

8. Marconi B, Bobyr I, Campanati A, Molinelli E, Consales V, Brisigotti $\mathrm{V}$, et al. Pseudoxanthoma elasticum and skin: Clinical manifestations, histopathology, pathomechanism, perspectives of treatment. Intractable Rare Dis Res. 2015;4(3):113-22. doi: 10.5582/irdr.2015.01014. [PubMed: 26361562]. [PubMed Central: PMC4561240]. 